\title{
Numerical convergence and stability of mixed formulation with X-FEM cut-off
}

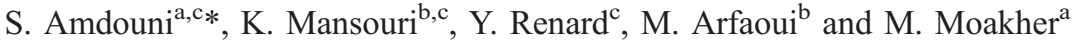 \\ ${ }^{a}$ LAMSIN-ENIT, Université Tunis El Manar, B.P. 37, 1002 Tunis-Belvédère, Tunisie; ${ }^{b}$ LGC-ENIT, \\ Université Tunis El Manar, B.P. 37, 1002 Tunis-Belvédère, Tunisie; ${ }^{c}$ Université de Lyon, CNRS, INSA- \\ Lyon, ICJ UMR5208, LaMCoS UMR5259, F-69621 Villeurbanne, France
}

\begin{abstract}
In this paper, we are concerned with the mathematical and numerical analysis of convergence and stability of the mixed formulation for incompressible elasticity in cracked domains. The objective is to extend the extended finite element method (X-FEM) cut-off analysis done in the case of compressible elasticity to the incompressible one. A mathematical proof of the inf-sup condition of the discrete mixed formulation with X-FEM is established for some enriched fields. We also give a mathematical result of quasi-optimal error estimate. Finally, we validate these results with numerical tests.

On s'interresse dans ce papier à l'analyse mathématique et numérique de la conver-gence et de la stabilité de la formulation mixte d'un problème d'élasticité incompressible dans un domaine fissuré. L'objectif est d'étendre l'étude faite sur la variante X-FEM cut-off, dans le cas de l'élasticité compressible, au comportement incompressible. Une preuve mathématique de la condition inf-sup de la formulation mixte discrète avec X-FEM est établie pour certains champs enrichis. Nous donnons également un résultat mathématique de la quasi-optimalité de l'estimation d'erreur. Enfin, nous validons ces résultats avec des tests numériques.
\end{abstract}

Keywords: X-FEM cut-off; mixed formulation; linear elasticity; error estimate

Mots-clés: X-FEM cut-off; formulation mixte; élasticité lineaire; estimation d'erreur

\section{Introduction}

The presence of a crack in a structure reveals two types of discontinuities: a strong discontinuity that requires an adapted mesh to the shape of the crack, hence the domain is meshed at each time step and a weak discontinuity that requires refinement at the crack tip. These two operations lead to a huge computational cost. In order to overcome these difficulties, we use the extended finite element method (X-FEM). This method allows to model cracks, material inclusions and holes on non-conforming meshes. It was introduced by Moës, Dolbow, and Belytschko (1999). It consists of enriching the basis of the classical finite element method by a step function along the crack line and by some non-smooth functions representing the asymptotic displacement around the crack tip. To obtain an optimal accuracy, Chahine, Labrode, and Renard (2008) introduced a new enrichment strategy: the so-called X-FEM cut-off. This enrichment strategy uses a cut-off function to locate the crack tip surface. In their work,

*Corresponding author. Email: Sabre.Amdouni@insa-lyon.fr 
Chahine et al. have shown that the X-FEM cut-off has an optimal convergence rate of order $h$ and that the conditioning of the stiffness matrix does not deteriorate. In this work, we extend the numerical results given by Chahine et al. (2008) to an incompressible isotropic linear plane elasticity problem in fracture mechanics. In particular, this formulation must satisfy the so-called inf-sup or "Ladyzhenskaya-Brezzi-Babuška" (LBB) condition.

\section{Model problem and discretisation}

Let $\Omega$ be a two-dimensional cracked domain, $\Gamma_{\mathrm{c}}$ denotes the crack and $\Gamma$ the boundary of $\Omega$. We assume that $\Gamma \backslash \Gamma_{\mathrm{c}}$ is partitioned into two parts: $\Gamma_{\mathrm{N}}$ where a Neumann surface force $t$ is applied and $\Gamma_{\mathrm{D}}$ where a Dirichlet condition $\mathbf{u}=\mathbf{0}$ is prescribed (see Figure 1). We assume that we have a traction-free condition on $\Gamma_{c}$. Let $f$ be the body force applied on $\Omega$. The equilibrium equation, constitutive law and boundary conditions are given by

$$
\begin{array}{ll}
-\operatorname{div} \sigma(\mathbf{u})=f, & \text { in } \Omega, \\
\sigma(\mathbf{u})=\lambda \operatorname{tr} \varepsilon(\mathbf{u}) I+2 \mu \varepsilon(\mathbf{u}), & \text { in } \Omega, \\
\mathbf{u}=\mathbf{0}, & \text { on } \Gamma_{\mathrm{D}}, \\
\sigma(\mathbf{u}) \cdot \mathbf{n}=\mathbf{t}, & \text { on } \Gamma_{\mathrm{N}}, \\
\sigma(\mathbf{u}) \cdot \mathbf{n}=\mathbf{0}, & \text { on } \Gamma_{\mathrm{c}} .
\end{array}
$$

with $\varepsilon(\mathbf{u})=\frac{1}{2}\left(\nabla \mathbf{u}+\nabla \mathbf{u}^{T}\right)$ and $n$ is the outside normal to the domain $\Omega$.

Let $V=\left\{v \in \mathbf{H}^{1}(\Omega)\right.$ with $\mathbf{u}=0$ on $\left.\Gamma_{\mathrm{D}}\right\} Q=\mathrm{L}^{2}(\Omega), \sigma^{\mathrm{d}}$ is the deviatoric part of $\sigma$ and $p$ the hydrostatic pressure. By a classical way, we find the weak mixed formulation (Brezzi \& Fortin, 1991)

$$
\begin{cases}\text { Find }(\mathbf{u}, p) \in(V, Q) \text { such that : } & \\ a(\mathbf{u}, \mathbf{v})-b(\mathbf{v}, p)=L(\mathbf{v}), & \forall \mathbf{v} \in \mathbf{V}, \\ b(\mathbf{u}, q)=0, & \forall q \in Q\end{cases}
$$

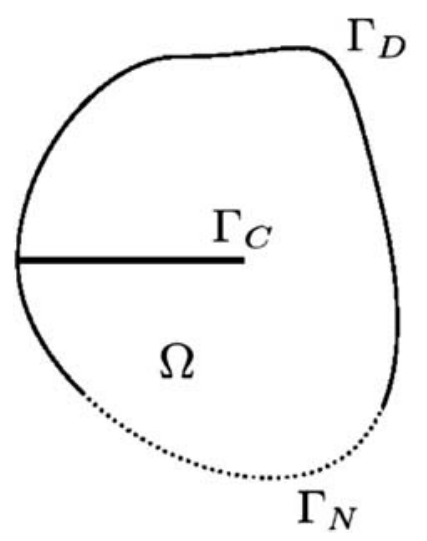

Figure 1. Cracked domains. 
with $a(\mathbf{u}, v)=\int_{\Omega} \sigma^{\mathrm{d}}(\mathbf{u}): \varepsilon(\mathbf{v}) d \Omega, b(v, p)=\int_{\Omega} p \operatorname{div} \mathbf{v} d \Omega, L(\mathbf{v})=\int_{\Omega} f \cdot \mathbf{v} d \Omega+\int_{\Gamma_{\mathrm{N}}} \mathbf{t} . \mathbf{v} d \Gamma$. Discretisation of the elasticity problem follows the usual steps. Let $\tau_{\mathrm{h}}$ be an affine mesh of the non-cracked domain $\bar{\Omega}$. We approximate $(\mathbf{u}, p)$ by $\left(\mathbf{u}_{\mathrm{h}}, p_{\mathrm{h}}\right) \in V_{\mathrm{h}} \times Q_{\mathrm{h}}$. The subspaces $V_{\mathrm{h}}$ and $Q_{\mathrm{h}}$ are finite dimensional spaces that will be defined later. The discretised problem is then:

$$
\begin{cases}\text { Find }\left(\mathbf{u}_{\mathrm{h}}, p_{\mathrm{h}}\right) \in\left(V_{\mathrm{h}}, Q_{\mathrm{h}}\right) \text { such that } & \\ a\left(\mathbf{u}_{\mathrm{h}}, \mathbf{v}_{\mathrm{h}}\right)-b\left(\mathbf{v}_{\mathrm{h}}, p_{\mathrm{h}}\right)=L\left(\mathbf{v}_{\mathrm{h}}\right), & \forall \mathbf{v}_{\mathrm{h}} \in V_{\mathrm{h}}, \\ b\left(\mathbf{u}_{\mathrm{h}}, q_{\mathrm{h}}\right)=0, & \forall q_{\mathrm{h}} \in Q_{\mathrm{h}}\end{cases}
$$

The existence of a stable finite element approximate solution $\left(\mathbf{u}_{\mathrm{h}}, p_{\mathrm{h}}\right)$ depends on choosing a pair of spaces $V_{\mathrm{h}}$ and $Q_{\mathrm{h}}$ such that the following LBB condition holds:

$$
\inf _{q_{\mathrm{h}} \in Q_{\mathrm{h}}} \sup _{\mathbf{v}_{\mathrm{h}} \in V_{\mathrm{h}}} \frac{b\left(\mathbf{v}_{\mathrm{h}}, q_{\mathrm{h}}\right)}{\left\|q_{\mathrm{h}}\right\|_{0, \Omega}\left\|\mathbf{v}_{\mathrm{h}}\right\|_{1, \Omega}} \geq \beta_{0},
$$

where $\beta_{0}>0$ is independent of $h$ (Brezzi \& Fortin, 1991). The satisfaction of this condition for a couple $\left(V_{\mathrm{h}}, Q_{\mathrm{h}}\right)$ is very difficult to prove in practical situations. Therefore, the numerical evaluation of the inf-sup has been widely used (Chapelle \& Bathe, 1993). It gives an indication of the verification of the LBB condition for a given finite element discretisation.

\section{X-FEM cut-off approximation spaces}

The idea of X-FEM is to use a classical finite element space enriched by some additional functions. These functions result from the product of global enrichment functions and some classical finite element functions. We consider the variant of X-FEM which uses a cut-off function to define the singular enrichment surface. The classical enrichment strategy for this problem is to use the asymptotic expansion of the displacement and pressure fields at the crack tip area. Indeed, the displacement is enriched by the Westergaard functions:

$$
F^{u}=\left\{F_{j}^{u}(x), 1 \leq j \leq 4\right\}=\left\{\sqrt{r} \sin \frac{\theta}{2}, \sqrt{r} \cos \frac{\theta}{2}, \sqrt{r} \sin \frac{\theta}{2} \sin \theta, \sqrt{r} \cos \frac{\theta}{2} \sin \theta\right\}
$$

where $(r, \theta)$ are polar coordinates around the crack's tip. These functions allow to generate the asymptotic non-smooth function at the crack's tip (Laborde, Renard, Pommier, \& Salaun, 2005). For the pressure, the asymptotic expansion at the crack tip is given by $p(r, \theta)=\frac{2 K_{I}}{3 \sqrt{2 \pi r}} \cos \frac{\theta}{2}+\frac{2 K_{I I}}{3 \sqrt{2 \pi r}} \sin \frac{\theta}{2}$ where $K_{\mathrm{I}}$ and $K_{\mathrm{II}}$ are the stress intensity factors. This expression is used to obtain the basis of enrichment of the pressure in the area of the crack's tip (Legrain, Moes, \& Huerta, 2008):

$$
F^{p}=\left\{F_{j}^{p}(x), 1 \leq j \leq 2\right\}=\left\{\frac{1}{\sqrt{r}} \cos \frac{\theta}{2} ; \frac{1}{\sqrt{r}} \sin \frac{\theta}{2}\right\} .
$$

The displacement and pressure are also enriched with a Heaviside-type function at the nodes for which the support of the corresponding shape functions is totally cut by the crack. Using this enrichment strategy, the discretisation spaces $V_{\mathrm{h}}$ and $Q_{\mathrm{h}}$ take the following forms: 


$$
\begin{aligned}
& V_{\mathrm{h}}=\left\{\mathbf{v}_{\mathrm{h}}=\sum_{i \in I} \alpha_{k} \psi_{u, k}+\sum_{i \in I_{\mathrm{H}}} \beta_{k} H \psi_{u, k}+\sum_{j=1}^{4} \gamma_{j} F_{j}^{u} \chi ; \quad \alpha_{k}, \beta_{k}, \gamma_{j} \in \mathbb{R}\right\}, \\
& Q_{\mathrm{h}}=\left\{p_{\mathrm{h}}=\sum_{i \in I} p_{i} \varphi_{p, i}+\sum_{i \in I_{\mathrm{H}}} b_{i}^{p} H \varphi_{p, i}+\sum_{j=1}^{2} c_{j}^{p} F_{j}^{p} \chi ; \quad p_{i}, b_{i}^{p}, c_{j}^{p} \in \mathbb{R}\right\},
\end{aligned}
$$

with $I$ the set of node indices of $\tau_{\mathrm{h}}, I_{\mathrm{H}}$ the set of node indices of $\tau_{\mathrm{h}}$ for which the supports of the shape functions are totally cut by the crack, $\varphi_{u, i}$ (resp. $\varphi_{p, i}$ ) being the scalar shape functions for displacement (resp. for pressure), $\psi_{u, k}$ being the vector shape functions defined by $\psi_{u, k}=\left\{\begin{array}{ll}\left(\begin{array}{c}\varphi_{u, i} \\ 0\end{array}\right) & \text { if } i=\frac{k+1}{2}, \\ \left(\begin{array}{c}0 \\ \varphi_{u, i}\end{array}\right) & \text { if } i=\frac{k}{2},\end{array}\right.$ and $\chi$ being a $\mathscr{C}^{1}$-piecewise function which is polynomial of degree 3 in the annular region $r_{0} \leq r \leq r_{1}$, and satisfies $\chi(r)=1$ if $r<r_{0}$ and $\chi(r)=0$ if $r>r_{1}$. In our case, we take $\chi(r)=\frac{2 r^{3}-3\left(r_{0}+r_{1}\right) r^{2}+6 r_{1} r_{0} r+\left(r_{0}-3 r_{1}\right) r_{0}^{2}}{\left(r_{0}-r_{1}\right)^{3}}$ if $r_{0} \leq r \leq r_{1}$ with $r_{0}=0.01$ and $r_{1}=0.49$.

\section{Proof of inf-sup condition and error analysis}

In this section, we prove that the LBB condition holds for the $P_{2} / P_{0}$ element without the singular enrichment of the pressure. In order to simplify the presentation, we assume that the crack cuts the mesh far enough from the vertices. We use a general technique introduced by Brezzi and Fortin (1991).

\subsection{Construction of a $\mathrm{H}_{1}$-stable interpolation operator}

The proof of the LBB condition requires the definition of an interpolation operator adapted to the proposed method. Since the displacement field is discontinuous across the crack on $\Omega$, we divide $\Omega$ into $\Omega_{1}$ and $\Omega_{2}$ according to the crack and a straight extension of it (Figure 2). Let $\mathbf{u}^{k}$ be the restriction of $\mathbf{u}$ to $\Omega_{k}, k \in\{1,2\}$. As $\mathbf{u} \in \mathbf{H}^{1}(\Omega)$, then there exists an extension $\tilde{\mathbf{u}}^{k}$ in $\mathbf{H}^{1}(\Omega)$ of $\mathbf{u}^{k}$ across the crack on $\Omega$ such that:

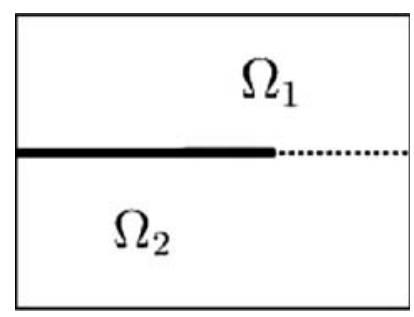

Figure 2. Domain decomposition. 


$$
\left\|\tilde{\mathbf{u}}^{k}\right\|_{1, \Omega} \leq C_{k}\left\|\mathbf{u}^{k}\right\|_{1, \Omega_{k}}
$$

where $C_{k}$ is independent of $\mathbf{u}$ (Adams, 1975).

Definition 1. Given a displacement field $\mathbf{u} \in \mathbf{H}_{0}^{1}(\Omega)$ and two extensions $\tilde{\mathbf{u}}^{1}$ and $\tilde{\mathbf{u}}^{2}$ of $\mathbf{u}^{1}$ and $\mathbf{u}^{2}$ in $\mathbf{H}_{0}^{1}(\Omega)$, respectively, we define $\Pi_{1} \mathbf{u}$ as the element of $V_{\mathrm{h}}$ such that:

$$
\Pi_{1} \mathbf{u}=\sum_{j \in I \backslash I_{\mathrm{H}}} \boldsymbol{\alpha}_{j} \varphi_{j}+\sum_{j \in I_{\mathrm{H}}}\left[\beta_{j} \varphi_{j} H_{1}+\gamma_{i} \varphi_{j} H_{2}\right]
$$

with

$$
\begin{gathered}
H_{1}(x)=\left\{\begin{array}{ll}
1 & \text { if } x \in \Omega_{1}, \\
0 & \text { if } x \in \Omega_{2},
\end{array} \quad H_{2}(x)=1-H_{1}(x),\right. \\
\alpha_{i}=\frac{1}{\left|\Delta_{i}\right|} \int_{\Delta_{i}} \tilde{\mathbf{u}}^{k} \mathrm{~d} x \quad \text { if } \quad x_{i} \in \Omega_{k}, \quad \beta_{i}=\frac{1}{\left|\Delta_{i}\right|} \int_{\Delta_{i}} \tilde{\mathbf{u}}^{1} \mathrm{dx},
\end{gathered}
$$

where $\Delta_{j}$ is the maximal ball centred at $x_{j}$ such that $\Delta_{j} \subset S_{j}$ and $\left\{x_{j}\right\}_{j=1}^{J}$ are the interior nodes of mesh $\tau_{\mathrm{h}}$.

This definition is inspired by the work of Chen and Nochetto (2000).

Lemma 1. The interpolation operator defined by (9) satisfies $\forall \mathbf{u} \in \mathbf{H}_{0}^{1}(\Omega)$

$$
\begin{gathered}
\left\|\Pi_{1} \mathbf{u}\right\|_{1, \Omega} \leq C\|\mathbf{u}\|_{1, \Omega}, \\
\left\|\mathbf{u}-\Pi_{1} \mathbf{u}\right\|_{r, \Omega} \leq C h^{1-r}\|\mathbf{u}\|_{1, \Omega}, \quad r=\{0,1\} .
\end{gathered}
$$

Proof. In the proof, we take $i \in\{1,2\}, k=3-i$ and $\tilde{s}$ the union of all elements surrounding the element $s$ of $\tau_{\mathrm{h}}$.

In order to prove this Lemma, we calculate the above estimates locally on every different type of triangles: non-enriched triangles, triangles cut by the straight extension of the crack, triangles partially enriched by the discontinuous functions, triangles containing the crack tip and triangles totally enriched by the discontinuous functions. Before, let us establish the following intermediary result:

Lemma 2. Let $\delta$ be a square of size $h$ centred at the crack tip (see Figure 3) and $f \in H_{0}^{1}\left(\delta \backslash \Gamma_{c}\right)$ with $f(x)=0, \forall x \in \tilde{\Gamma}_{c} \bigcap \delta$ (where $\tilde{\Gamma}_{c}$ is the extension of the crack $\left.\Gamma_{c}\right)$. Then, there exists $c>0$, independent of $h$ such that:

$$
\|f\|_{0, \delta} \leq c h\|\nabla f\|_{0, \delta \backslash \Gamma_{c}} .
$$




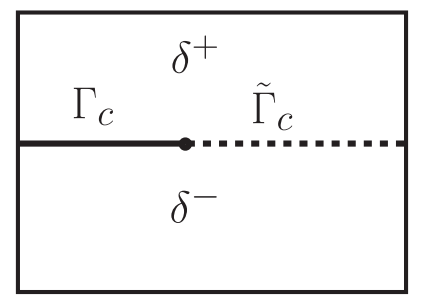

Figure 3. Centred domain on the crack tip.

Proof. Dividing the square into two parts $\delta^{+}$(above the crack) and $\delta^{-}$(below the crack). Let $\hat{f}^{+}=f \circ T_{k}$ defined on the reference rectangle $\hat{\delta}^{+}$(assumed of size 1) obtained by an affine transformation $T_{K}$ of the rectangle $\delta^{+}$. Then, by construction, $\hat{f}^{+}(x)=$ $0 \forall x \in\left\{x_{1} \geq 0\right\} \times\left\{x_{2}=0\right\}$ which implies the following Poincaré inequality:

$$
\left\|\hat{f}^{+}\right\|_{0, \hat{\delta}^{+}} \leq c\left\|\nabla \hat{f}^{+}\right\|_{0, \hat{\delta}^{+}}
$$

Using inequality (13) and the fact that the mesh is affine, we obtain:

$$
\begin{gathered}
\|f\|_{0, \delta^{+}} \leq c\left|\operatorname{det}\left(J_{K}\right)\right|^{1 / 2}\left\|\hat{f}^{+}\right\|_{0, \delta^{+}} \leq c\left|\operatorname{det}\left(J_{K}\right)\right|^{1 / 2}\left\|\nabla \hat{f}^{+}\right\|_{0, \hat{\delta}^{+}} \leq c\left|\operatorname{det}\left(J_{K}\right)\right|^{-1 / 2} \\
\left\|J_{K}\right\|_{2}\left|\operatorname{det}\left(J_{K}\right)\right|^{1 / 2}|f|_{1, \delta^{+}} \leq c h|f|_{1, \delta^{+}},
\end{gathered}
$$

where $|\cdot|_{1, \delta^{+}}$is the $\mathbf{H}^{1}$ semi-norm on $\delta^{+}$. Thus

$$
\|f\|_{0, \delta^{+}} \leq c h|f|_{1, \delta^{+}}
$$

Similarly, we prove the same result for $\delta^{-}$which finish the proof of Lemma 2.

\subsubsection{Non-enriched triangles}

Let $s$ be a non-enriched triangle in $\Omega_{i}$. In this case, we have $\Pi_{1} \mathbf{u}=\Pi_{1} \tilde{\mathbf{u}}^{i}$ on $\Omega_{i}$. Because $\tilde{\mathbf{u}}^{i}$ is continuous over $\Omega$, this operator is equivalent to the classical operator of Chen and Nochetto (2000). Then, we have

$$
\left\|\Pi_{1} \mathbf{u}\right\|_{1, s}=\left\|\Pi_{1} \tilde{\mathbf{u}}^{i}\right\|_{1, s} \leq c\left\|\tilde{\mathbf{u}}^{i}\right\|_{1, \tilde{s}}
$$

and

$$
\left\|\mathbf{u}-\Pi_{1} \mathbf{u}\right\|_{r, s}=\left\|\mathbf{u}^{i}-\Pi_{1} \tilde{\mathbf{u}}^{i}\right\|_{r, s}=\left\|\tilde{\mathbf{u}}^{i}-\right\| \Pi_{1} \tilde{\mathbf{u}}^{i}\left\|_{r, s} \leq c h^{1-r}\right\| \tilde{\mathbf{u}}^{i} \|_{1, \tilde{s}}
$$

\subsubsection{Triangles cut by the straight extension of the crack or containing the crack tip}

Let $s$ be a triangle cut by the straight extension of the crack or containing the crack tip (see Figure 4(c)). Then $\Pi_{1} \mathbf{u}=\alpha_{1} \varphi_{1}+\alpha_{2} \varphi_{2}+\alpha_{3} \varphi_{3} \quad$ on $\quad s$, with: $\quad \boldsymbol{\alpha}_{1}=\frac{1}{\left|\Delta_{1}\right|} \int_{\Delta_{1}} \tilde{\mathbf{u}}^{1} \mathrm{~d} x, \boldsymbol{\alpha}_{2}=$ $\frac{1}{\left|\Delta_{2}\right|} \int_{\Delta_{2}} \tilde{\mathbf{u}}^{2} \mathrm{~d} x$ and $\alpha_{3}=\frac{1}{\left|\Delta_{3}\right|} \int_{\Delta_{3}} \tilde{\mathbf{u}}^{2} \mathrm{~d} x$. 


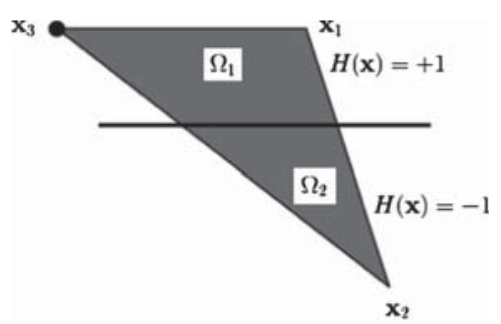

(a) A triangle partially enriched by the discontinuous functions

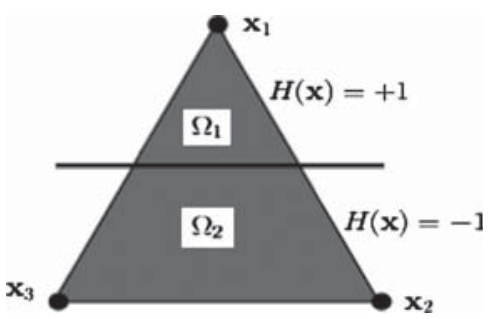

(b) A triangle totally enriched by the discontinuous functions

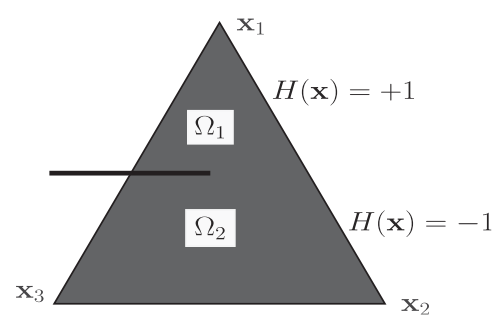

(c) A triangle containing the crack tip

Figure 4. The different types of enriched triangles. The enrichment with the Heaviside function are marked with a bullet.

We remark that:

$$
\Pi_{1} \mathbf{u}=\tilde{\boldsymbol{\alpha}}_{1} \varphi_{1}+\boldsymbol{\alpha}_{2} \varphi_{2}+\boldsymbol{\alpha}_{3} \varphi_{3}+\left(\boldsymbol{\alpha}_{1}-\tilde{\boldsymbol{\alpha}}_{1}\right) \varphi_{1}=\Pi_{1} \tilde{\mathbf{u}}^{2}+\left(\boldsymbol{\alpha}_{1}-\tilde{\boldsymbol{\alpha}}_{1}\right) \varphi_{1},
$$

with $\tilde{\alpha}_{1}=\frac{1}{\left|\Delta_{1}\right|} \int_{\Delta_{1}} \tilde{\mathbf{u}}^{2} \mathrm{~d} x$. By the triangle inequality, we may write

$$
\begin{gathered}
\left\|\Pi_{1} \mathbf{u}\right\|_{1, s} \leq\left\|\Pi_{1} \tilde{\mathbf{u}}^{2}\right\|_{1, s}+\left|\boldsymbol{\alpha}_{1}-\tilde{\boldsymbol{\alpha}}_{1}\right|\left\|\varphi_{1}\right\|_{1, s}, \\
\left\|\mathbf{u}-\Pi_{1} \mathbf{u}\right\|_{r, s} \leq\left\|\mathbf{u}-\Pi_{1} \tilde{\mathbf{u}}^{2}\right\|_{r, s}+\left|\boldsymbol{\alpha}_{1}-\tilde{\alpha}_{1}\right|\left\|\varphi_{1}\right\|_{r, s} \leq\left\|\mathbf{u}-\tilde{\mathbf{u}}^{2}\right\|_{r, s}+ \\
\left\|\tilde{\mathbf{u}}^{2}-\Pi_{1} \tilde{\mathbf{u}}^{2}\right\|_{r, s}+\left|\boldsymbol{\alpha}_{1}-\tilde{\boldsymbol{\alpha}}_{1}\right|\left\|\varphi_{1}\right\|_{r, s},
\end{gathered}
$$

where $\left\|\varphi_{1}\right\|_{r, s} \leq c h^{1-r} \quad$ because $\quad \varphi_{1} \quad$ is the piecewise $P_{1}$ basis function, $\left\|\Pi_{1} \tilde{\mathbf{u}}^{2}\right\|_{r, s} \leq c h^{1-r}\left\|\tilde{\mathbf{u}}^{2}\right\|_{1, \tilde{s}}$ because $\tilde{\mathbf{u}}^{k}$ is continuous over $\Omega, \quad\left\|\mathbf{u}-\tilde{\mathbf{u}}^{2}\right\|_{0, s} \leq c h \|$ $\mathbf{u}-\tilde{\mathbf{u}}^{2} \|_{1, \delta}$, and if we use Cauchy-Schwartz inequality and Lemma 2, we obtain

$$
\left|\alpha_{1}-\tilde{\alpha}_{1}\right| \leq \frac{h}{\left|\Delta_{1}\right|}\left\|\tilde{\mathbf{u}}^{1}-\tilde{\mathbf{u}}^{2}\right\|_{0, \Delta_{1}} \leq c \frac{h^{2}}{\left|\Delta_{1}\right|}\left\|\nabla\left(\tilde{\mathbf{u}}^{1}-\tilde{\mathbf{u}}^{2}\right)\right\|_{0, \delta} .
$$

Therefore,

$$
\begin{gathered}
\left\|\Pi_{1} \mathbf{u}\right\|_{1, s} \leq c\left(\left\|\tilde{\mathbf{u}}^{2}\right\|_{1, \tilde{s}}+\left\|\tilde{\mathbf{u}}^{1}-\tilde{\mathbf{u}}^{2}\right\|_{1, \delta}\right), \\
\left\|\mathbf{u}-\Pi_{1} \mathbf{u}\right\|_{r, s} \leq c h^{1-r}\left(\left\|\mathbf{u}-\tilde{\mathbf{u}}^{2}\right\|_{1, s}+\left\|\tilde{\mathbf{u}}^{2}\right\|_{1, \tilde{s}}+\left\|\tilde{\mathbf{u}}^{1}-\tilde{\mathbf{u}}^{2}\right\|_{1, \delta}\right) .
\end{gathered}
$$




\subsubsection{Triangles partially enriched by the discontinuous functions}

Let $s$ be a triangle partially enriched by the discontinuous functions (see Figure 4(a)). In this case, we have $\Pi_{1} \mathbf{u}=\Pi_{1} \tilde{\mathbf{u}}_{1}+\left(\alpha_{2}-\tilde{\alpha}_{2}\right) \varphi_{2}$ on $s \cap \Omega_{1}$ and $\Pi_{1} u=\Pi_{1} \tilde{\mathbf{u}}_{2}+\left(\alpha_{1}-\tilde{\alpha}_{1}\right) \varphi_{1}$ on $s \cap$ $\Omega_{2}$ with $\tilde{\alpha_{1}}=\frac{1}{\left|\Delta_{1}\right|} \int_{\Delta_{1}} \tilde{\mathbf{u}}^{2} \mathrm{~d} x$ and $\alpha_{2}=\frac{1}{\left|\Delta_{2}\right|} \int_{\Delta_{2}} \tilde{\mathbf{u}}^{1} \mathrm{~d} x$.

In the same manner, we prove that

$$
\begin{gathered}
\left\|\Pi_{1} \mathbf{u}\right\|_{1, s \cap \Omega_{i}} \leq c\left(\left\|\tilde{\mathbf{u}}_{i}\right\|_{1, \tilde{s}}+\left\|\tilde{\mathbf{u}}^{k}-\tilde{\mathbf{u}}^{i}\right\|_{1, \delta}\right), \\
\left\|\mathbf{u}-\Pi_{1} \mathbf{u}\right\|_{r, s \cap \Omega_{i}} \leq c h^{1-r}\left(\left\|\tilde{\mathbf{u}}^{i}\right\|_{1, \tilde{s}}+\left\|\tilde{\mathbf{u}}^{k}-\tilde{\mathbf{u}}^{i}\right\|_{1, \delta}\right) .
\end{gathered}
$$

\subsubsection{Triangles totally enriched by the discontinuous functions}

Let $s$ be the triangle totally enriched by the discontinuous functions (see Figure 4(b)). In this case, we have: $\Pi_{1} \mathbf{u}=\Pi_{1} \widetilde{\mathbf{u}}^{i}$ on $s \cap \Omega_{i}$. Then, we have

$$
\begin{gathered}
\left\|\Pi_{1} \mathbf{u}\right\|_{1, s \cap \Omega_{i}} \leq\left\|\Pi_{1} \tilde{\mathbf{u}}^{i}\right\|_{1, s}, \\
\left\|\mathbf{u}-\Pi_{1} \mathbf{u}\right\|_{r, s \cap \Omega_{i}} \leq\left\|\tilde{\mathbf{u}}^{i}-\Pi \tilde{\mathbf{u}}^{i}\right\|_{1, s} \leq c h^{1-r}\left\|\tilde{\mathbf{u}}^{i}\right\|_{r, \tilde{s}} .
\end{gathered}
$$

Inequalities (15), (17), (19) and (21) imply the first inequality of Lemma 1. Inequalities (16), (18), (20) and (22) imply the second and third inequalities of Lemma 1.

\subsection{Construction of a local interpolation operator}

In this subsection, we prove the discrete inf-sup condition for the $P_{2} / P_{0}$ element with the additional assumption, that the crack cuts the mesh far enough from the nodes.

Definition 2. Let $\mathbf{u} \in \mathbf{H}^{1}(\Omega)$. We define $\Pi_{2} u$ as the element of $V_{\mathrm{h}}$ such that

$$
\Pi_{2} \mathbf{u}=\sum_{k \in \tau_{\mathrm{h}} / \tau_{\mathrm{H}}} \sum_{i=1}^{3} \alpha_{i} \varphi_{i}+\sum_{k \in \tau_{\mathrm{H}}} \sum_{i=1}^{3}\left(\beta_{i} \varphi_{i} H_{1}+\gamma_{i} \varphi_{i} H_{2}\right)
$$

where $\tau_{\mathrm{H}}$ is the set of triangle totally cut by the crack, $\varphi_{i}$ is the classical finite element shape function of order 2 associated to node $i$ being the centre of the edge $e_{i}$ of the element $\mathrm{K}$ and with

$$
\alpha_{i}=\frac{\int_{e_{i}} \mathbf{u}}{\int_{e_{i}} \varphi_{i}}, \quad \beta_{i}=\frac{\int_{e_{i} \cap \Omega_{1}} \mathbf{u}}{\int_{e_{i} \cap \Omega_{1}} \varphi_{i}}, \quad \gamma_{i}=\frac{\int_{e_{i} \cap \Omega_{2}} \mathbf{u}}{\int_{e_{i} \cap \Omega_{2}} \varphi_{i}} .
$$

Lemma 3. Suppose that the crack cuts the mesh far enough from the nodes, then the interpolation operator defined by (23) satisfies $\forall \mathbf{u} \in V_{\mathrm{h}}$

$$
\int_{s \backslash \Gamma_{c}} \operatorname{div}\left(\mathbf{u}-\Pi_{2} \mathbf{u}\right)=0 \quad \forall s \in \tau_{\mathrm{h}},
$$




$$
\left\|\Pi_{2} \mathbf{u}\right\|_{1, s \cap \Omega_{i}} \leq c\left(h^{-1}\left\|\tilde{\mathbf{u}}^{i}\right\|_{0, s}+\left|\tilde{\mathbf{u}}^{i}\right|_{1, s}\right) \quad \forall s \in \tau_{\mathrm{h}} .
$$

Therefore, the discrete inf-sup condition for the $\mathrm{P}_{2} / \mathrm{P}_{0}$ element holds.

Proof. The first equation is obvious. Now, let $s$ be a triangle totally cut by the crack. Then by using triangle inequality, the hypothesis "crack far enough from nodes" and Cauchy-Schwarz inequality, we have:

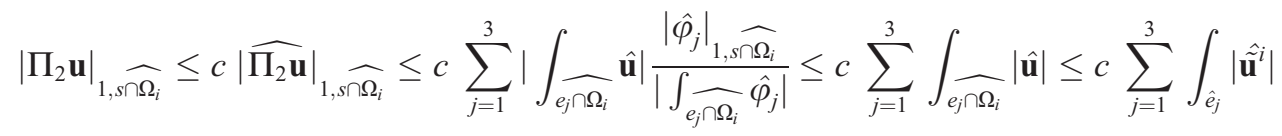

$$
\begin{aligned}
& \leq c \quad\left\|\hat{\tilde{\mathbf{u}}}^{i}\right\|_{1, \hat{s}}
\end{aligned}
$$

and by a scaling argument, we have:

$$
\left\|\Pi_{2} \mathbf{u}\right\|_{1, s \cap \Omega_{i}} \leq c\left(h^{-1}\left\|\tilde{\mathbf{u}}^{i}\right\|_{0, s}+\left|\tilde{\mathbf{u}}^{i}\right|_{1, s}\right) .
$$

Now for non-enriched triangle, we use the same argument to prove:

$$
\left\|\Pi_{2} \mathbf{u}\right\|_{1, s} \leq c\left(h^{-1}\|\mathbf{u}\|_{0, s}+|\mathbf{u}|_{1, s}\right),
$$

which finishes the proof of Lemma 3.

\subsection{Error analysis}

We suppose in this section that the non-cracked domain $\bar{\Omega}$ has a regular boundary, and that $f$, $t$ are smooth enough, for the solution $(\mathbf{u}, p)$ of the mixed elasticity problem to be written as a sum of a singular part $\left(\mathbf{u}_{\mathrm{s}}, p_{\mathrm{s}}\right)$ and a regular part $\left(\mathbf{u}-\mathbf{u}_{s}, p-p_{s}\right)$ in $\Omega$ satisfying $\mathbf{u}-\mathbf{u}_{s} \in \mathbf{H}^{2}$ and $p-p_{s} \in \mathbf{H}^{1}$.

Proposition 1. Under the assumption of existence and uniqueness of solutions $(\mathbf{u}, \mathrm{p})$ and $\left(\mathbf{u}_{\mathrm{h}}\right.$, $\mathrm{p}_{\mathrm{h}}$ ) of the continuous (6) and discrete (7) mixed elasticity problems, and if the LBB condition is satisfied, then:

$$
\left\|\mathbf{u}-\mathbf{u}_{\mathrm{h}}\right\|_{1, \Omega}+\left\|p-p_{\mathrm{h}}\right\|_{0, \Omega} \leq c h\left[\left\|\mathbf{u}-\chi \mathbf{u}_{s}\right\|_{2, \Omega}+\left\|p-\chi p_{s}\right\|_{1, \Omega}\right]
$$

where $\chi$ is the cut-off function.

Proof. By using the equivalent Céa lemma (see Brezzi \& Fortin, 1991), we have $\forall \mathbf{v}_{\mathrm{h}} \in V^{h}$ and $q_{h} \in Q^{h}$ :

$$
\left\|\mathbf{u}-\mathbf{u}_{\mathrm{h}}\right\|_{1, \Omega}+\left\|p-p_{\mathrm{h}}\right\|_{0, \Omega} \leq c\left[\left\|\mathbf{u}-\mathbf{v}_{\mathrm{h}}\right\|_{1, \Omega}+\left\|p-q_{\mathrm{h}}\right\|_{0, \Omega}\right] .
$$

Now let $\Pi_{\mathrm{h}} \mathbf{u}$ be the classical interpolation operator introduced by Nicaise, Renard, and Chahine (2011), then we have: 


$$
\left\|\mathbf{u}-\Pi_{\mathrm{h}} \mathbf{u}\right\|_{1, \Omega} \leq c h\left\|\mathbf{u}-\chi \mathbf{u}_{s}\right\|_{2, \Omega} .
$$

Let $\Pi_{\mathrm{h}} p=\Pi_{1} p+\sum_{i=1}^{2} c_{i} F_{i p} \chi=\Pi_{1} p+\chi p_{s}$, where $\Pi_{1}$ is the interpolation operator defined in Section 4.1. Then:

$$
\left\|p-\Pi_{\mathrm{h}} p\right\|_{0, \Omega}=\left\|p_{r}-\Pi_{1} p_{r}\right\|_{0, \Omega} \leq c h\left\|p_{r}\right\|_{1, \Omega} .
$$

Finally, the result of Proposition 1 can be obtained by choosing $\mathbf{v}_{\mathrm{h}}=\Pi_{\mathrm{h}} \mathbf{u}$ and $q_{\mathrm{h}}=\Pi_{\mathrm{h}} p$ in (26) and by using Equations (27) and (28).

\section{Numerical study}

The numerical tests are made on a non-cracked domain defined by $\bar{\Omega}=$ ]$-0.5,0.5[\times]-0.5,0.5\left[\right.$, and the considered crack is the line segment $\left.\Gamma_{c}=\right]-0.5 ; 0[\times\{0\}$ (see Figure 5(a)). To remove rigid body motions, we eliminate three degrees of freedom (see Figure 5(a)). In this numerical test, we impose only a boundary condition of Neumann type (see Figure 5(a)), in order to avoid possibility of singular stress for mixed Dirichlet-Neumann condition at transition points. The finite element method is defined on a structured triangulation of $\bar{\Omega}$. The von Mises stress for this test is presented in Figure 6(b). As expected the von Mises stress is concentrated at the crack tip. The notation $P_{i}$ (resp. $P_{i}^{+}$) means that we use an extended finite element method of order $i$ (resp. with an additional cubic buble function) and $P_{j}$ disc means that we use a discontinuous extended finite element method. The reference solution is obtained with a structured $P_{1} / P_{2}$ method and $h=1 / 160$.

\subsection{Numerical inf-sup test}

In this section, we numerically study the inf-sup condition and its dependence on the position of the crack. First, the inf-sup condition is evaluated using gradually refined structured triangulation meshes. The evolution of the numerical inf-sup value is plotted in Figure 6(a) with respect to the element size. From this figure, we can conclude that the numerical inf-sup value is stable for all studied formulations. Let $\delta$ be the crack position as shown in Figure 5 (b). To test the influence of the position of the crack on the inf-sup condition, we check the LBB condition by decreasing $\delta$. The tests are made, on a $P_{1}^{+} / P_{1}$ formulation, with $h=1 / 100$ (see Figure 7(a)) and $h=1 / 10$ (see Figure 7(b)). The results presented in Figures 7 (a) and (b) show that the inf-sup condition remains bounded regardless of the position of the crack. Hence, one can conclude that the formulation is stable independently of the position of the crack.

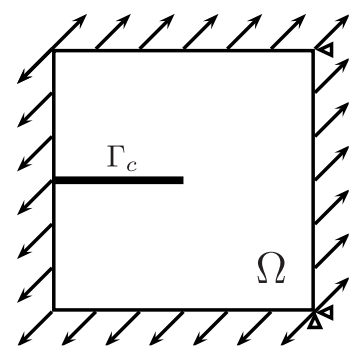

(a) Cracked specimen

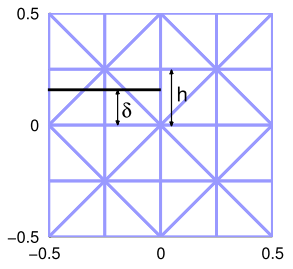

(b) Position of the crack.

Figure 5. Cracked specimen and position of the crack. 


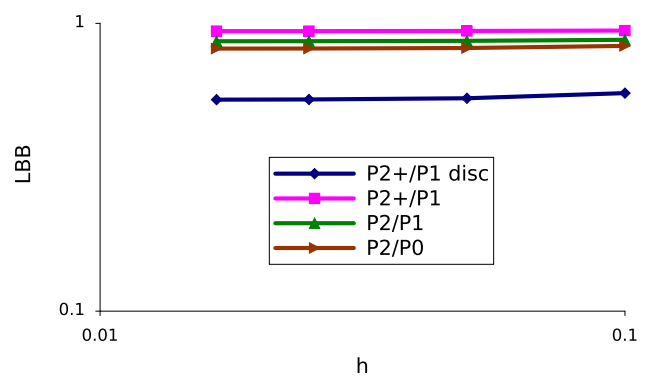

(a) Evaluation of the inf-sup condition

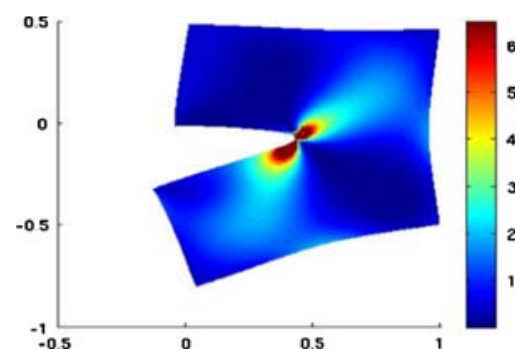

(b) von Mises stress

Figure 6. Evolution of the inf-sup condition for mixed problem and von Mises stress $(\delta=0)$.

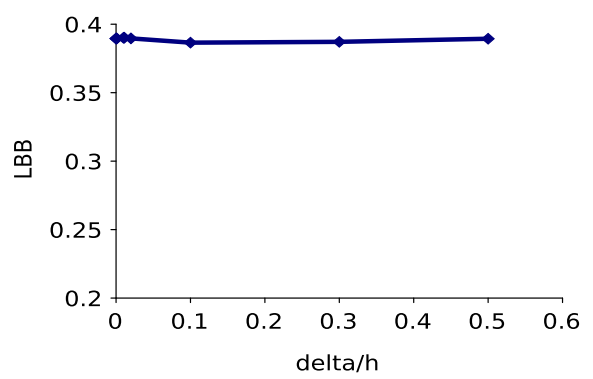

(a) $h=1 / 10$

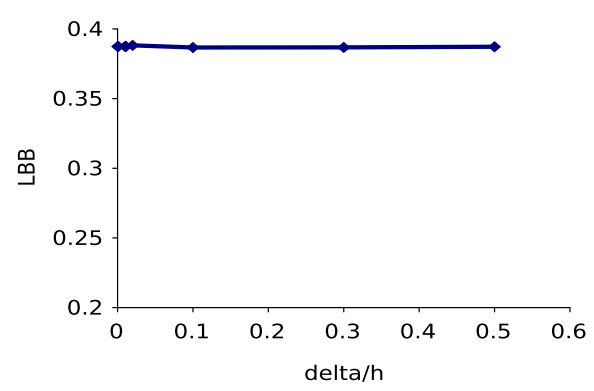

(b) $h=1 / 100$

Figure 7. Evolution of the inf-sup condition as a function of the position of the crack.

\subsection{Convergence rate and the computational cost}

Figures 8(a)-(c) show a comparison between the convergence rates of the X-FEM fixed area and X-FEM cut-off for the $\mathbf{L}^{2}$-norm and $\mathbf{H}^{1}$-norm $\left(P 1^{+} / P_{1}\right.$ element are used). These errors were obtained by running the test problem for some values of the parameter $n_{s}$, where $n_{s}$ is the number of subdivision (number of cells) in each direction $h=\frac{1}{n_{s}}$. Figure 8 (b) confirms that the convergence rate for the energy norm is of order $h$ for both variants of the X-FEM: with fixed area and cut-off. Figure 8(a) shows that the convergence rates for the $\mathbf{L}^{2}$-norm in displacement are of order $h^{2}$ for both variants. Figure 8(c) shows that the convergence rates for the $\mathbf{L}^{2}$-norm in pressure are $h$ for both variants. Compared to the X-FEM method with a fixed enrichment area, the convergence rate for X-FEM cut-off is very close but the error values are a bit larger. In order to test the computational cost of X-FEM cut-off, Table 1 shows a comparison between the number of degrees of freedom for different refinements of the classical method X-FEM with fixed enrichment area and the cut-off method. This latter enrichment leads to a significant decrease in the number of degrees of freedom. The condition number of the linear system associated to the cut-off enrichment is much better than the one associated with the X-FEM with a fixed enrichment area (see Figure 9). We can conclude that, similar to the X-FEM with fixed enrichment area, the X-FEM cut-off leads to an optimal convergence rate and also reduces the approximation errors but without significant additional costs.

The numerical tests of the higher order X-FEM method $\left(P_{2}^{+} / P_{1} \operatorname{disc}, P_{2}^{+} / P_{1}, P_{2} / P_{1}\right.$ and $P_{2} / P_{0}$ ) do not give an optimal order of convergence (see Figures 10(a)-(d)). This means that 


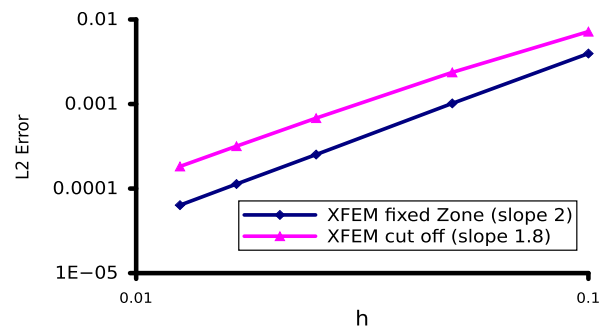

(a) $\mathbf{L}$-displacement error

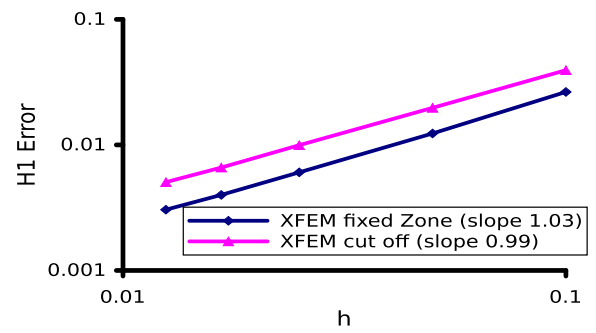

(b) H1-displacement error

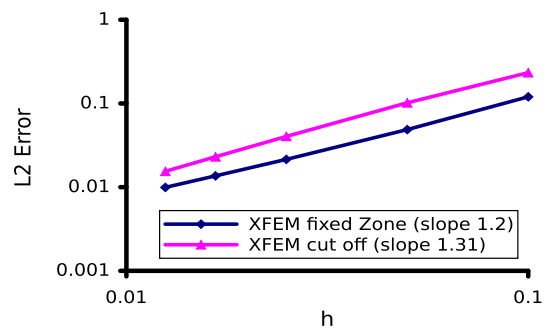

(c) $L^{2}$-pressure error

Figure 8. Errors for the mixed problem with enriched $P_{1}^{+} / P_{1}$ elements.

Table 1. Number of degrees of freedom for enriched $P_{2} / P_{1}$ element.

\begin{tabular}{lcc}
\hline & \multicolumn{2}{c}{ Number of degrees of freedom } \\
\cline { 2 - 3 } Number of cells in each direction & X-FEM fixed enrichment area & X-FEM cut-off \\
\hline 40 & 13456 & 11516 \\
60 & 30046 & 25666 \\
80 & 53376 & 45416 \\
\hline
\end{tabular}

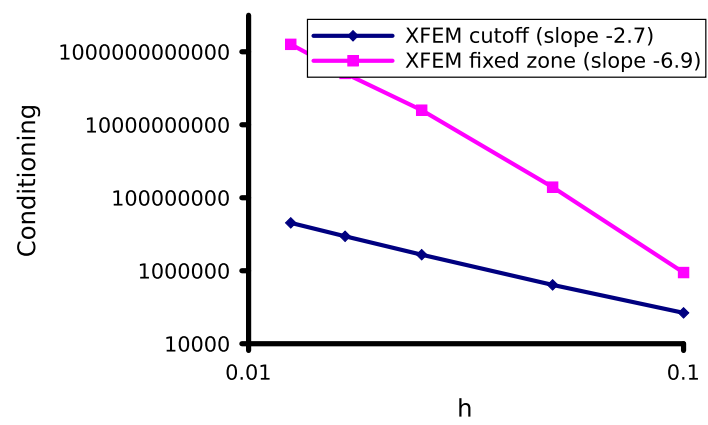

Figure 9. Conditioning number of the stiffness matrix for enriched $P_{2} / P_{1}$ element.

the enrichment function does not capture the behaviour of the solution at the crack's tip. This result was expected as the asymptotic displacement at the crack tip belongs to $\mathbf{H}^{3 / 2-\eta}(\Omega)$ for 


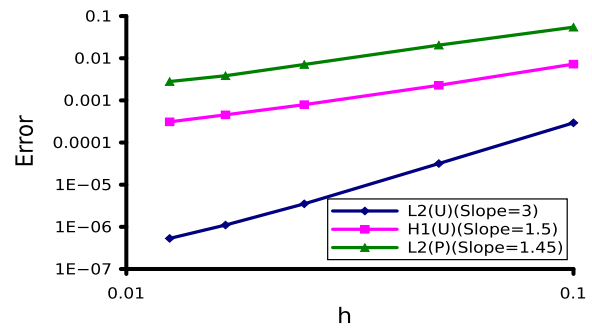

(a) Enriched $P_{2}^{+} / P_{1}$ disc element

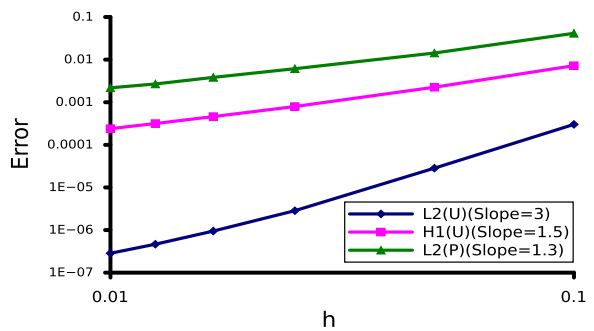

(c) Enriched $P_{2} / P_{1}$ element

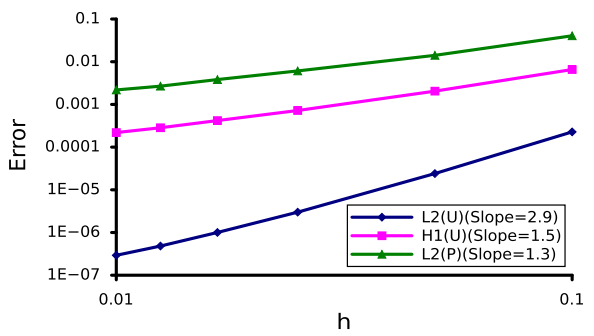

(b) Enriched $P_{2}^{+} / P_{1}$ element

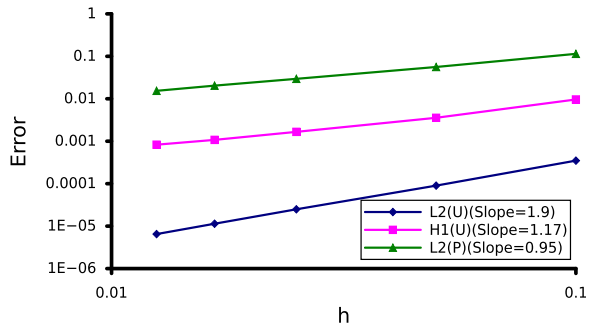

(d) Enriched $P_{2} / P_{0}$ element

Figure 10. Convergence rate for the high-order elements (logarithmic scales).

all $\eta>0$. Then, for the X-FEM cut-off, the convergence rate remains limited to $h^{3 / 2}$ with high-order polynomials. To have an optimal convergence rate, one must make an asymptotic expansion of order 2 to find the correct expression of the enrichment basis for the displacement and pressure.

\section{Conclusion}

From this study, we can conclude that the X-FEM cut-off mixed formulation is stable, regardless of the position of the crack. Similarly, to the X-FEM with fixed enrichment area, the XFEM cut-off gives an optimal convergence rate but without significant additional costs. For shape functions of higher order, the convergence rate is limited to $h^{3 / 2}$. This result was expected as the main singularity belongs to $\mathbf{H}^{5 / 2-\eta}(\Omega)$ for all $\eta>0$.

\section{References}

Adams, R., Sobolev spaces, New York: Academic Press, 1975.

Brezzi, F., \& Fortin, M. (1991). Mixed and hybrid finite element methods (Vol. 15). New York: Springer Series in Computational Mathematics.

Chahine, E., Labrode, P., \& Renard, Y. (2008). Crack tip enrichment in the X-FEM method using a cutoff function. International Journal for Numerical Method in Engineering, 75(6), 629-646.

Chapelle, D., \& Bathe, K.J. (1993). The inf-sup test. Computers and Structures, 47(4-5), 537-545.

Chen, Z., \& Nochetto, R.H. (2000). Residual-type a posteriori error estimates for elliptic obstacle problems. Numerische Mathematik, 84, 527-548.

Laborde, P., Renard, Y., Pommier, J., \& Salaun, M. (2005). High order extended finite element method for cracked domains. International Journal for Numerical Method in Engineering, 64, 354-381.

Legrain, G., Moes, N., \& Huerta, A. (2008). Stability of incompressible formulations enriched with XFEM. Computer Methods in Applied Mechanics and Engineering, 197, 1835-1849. 
Moës, N., Dolbow, J., \& Belytschko, T. (1999). A finite element method for crack growth without remeshing. International Journal for Numerical Methods in Engineering, 46, 131-150.

Nicaise, S., Renard, Y., \& Chahine, E. (2011). Optimal convergence analysis for the extended finite element method. International Journal for Numerical Methods in Engineering, 86, 528-548. 